\title{
FROZEN COMPLEMENT IN THE WASSERMANN REACTION
}

\author{
E. H. RUEDIGER
}

From the Pathological Latoratory of the Bismarck Hospital, Bismarck, N. D.

Without giving any details, Moledezky says frozen complement retains its strength indefinitely. As complement usually deteriorates within 3 days it is the most expensive ingredient in the Wassermann reaction, and as the time for standardization is short a method by which complement can be preserved is very desirable. Other methods having been found unsatisfactory frozen complement was studied.

\section{TEST 1}

Complement serums $1,2,3,5,6$ and 7 , each composed of the pooled serums of 3 guinea-pigs, were divided into two portions, A and B. Portion A of each number was tested on 4 human serums within 24 hours after having been secured and without having been frozen. Portion $B$ of each number was frozen within 24 hours after having been secured, it was kept frozen and at intervals of a week portion $B$ of each number was tested on the same human serums that had been used in testing portion A. Each mixiure of complement was kept under observation for a period of 4 weeks, at the end of which time the human serums were again tested with fresh complements 4 and 8 in order to detect any change in the human serums should there be such.

Table 1 shows the results obtained with mixed frozen complements. Frozen complement a week old usually was fixed a little better than was the fresh complement of the same number. With frozen complement two weeks old the results were almost identical with those obtained with the fresh complement. After two weeks fixability and hemolysis became poorer which was not due to changes in the human serums as is shown in the tests with control complements 4 and 8 . Complements 5, 6 and 7 deteriorated more rapidly than did complements 1,2 and 3 , and was brought about by mild weather.

\section{TEST 2}

Complements $9,10,11,13,14$ and 15 were unmixed, each was composed of the serum from one guinea-pig. Each complement serum was divided into two portions, A and B. Portion A of each number was tested on four human

Received for publication May 15, 1919.

1 Jour. Am. Med. Assn., 1918, 71, p. 968. 
TABLE 1

Mixed Frozen Complement Tested at Intervals of a WeEx

\begin{tabular}{|c|c|c|c|c|c|c|c|c|c|c|c|c|}
\hline \multirow{3}{*}{$\begin{array}{l}\text { Number } \\
\text { of } \\
\text { Comple- } \\
\text { ment }\end{array}$} & \multirow{3}{*}{$\begin{array}{c}\text { Portions } \\
\text { A-Fresh } \\
\text { B-Frozen }\end{array}$} & \multirow{3}{*}{$\begin{array}{c}\text { Age, } \\
\text { Weeks }\end{array}$} & \multirow{3}{*}{$\begin{array}{c}\text { Number } \\
\text { of } \\
\text { Serum }\end{array}$} & \multirow{3}{*}{$\begin{array}{l}\text { Dilu- } \\
\text { tion } \\
\text { of } \\
\text { Ambo- } \\
\text { ceptor }\end{array}$} & \multicolumn{6}{|c|}{ Readings* } & \multirow{3}{*}{\multicolumn{2}{|c|}{ Results }} \\
\hline & & & & & \multicolumn{3}{|c|}{$\begin{array}{c}\text { Autigen } \\
\text { Tubes }\end{array}$} & \multicolumn{3}{|c|}{$\begin{array}{l}\text { Control } \\
\text { Tubes }\end{array}$} & & \\
\hline & & & & & 1 & 2 & 3 & $1^{\prime}$ & $2^{\prime \prime}$ & $3^{\prime}$ & & \\
\hline 1 & $A$ & 0 & $\begin{array}{l}1 \\
2 \\
3 \\
3 \\
4\end{array}$ & $1: 400$ & $\begin{array}{l}\operatorname{tr} \\
\pm \\
+ \\
+\end{array}$ & $\begin{array}{l}0 \\
0 \\
0 \\
+\end{array}$ & $\begin{array}{c}0 \\
0 \\
0 \\
\pm\end{array}$ & $\begin{array}{l}+ \\
+ \\
+\end{array}$ & $\begin{array}{l}+ \\
+ \\
+ \\
+\end{array}$ & $\begin{array}{l} \pm \\
\pm \\
\pm\end{array}$ & $\begin{array}{l}\text { Strongly positive, } \\
\text { Strongly positive, } \\
\text { Strongly positive, } \\
\text { Negative, }\end{array}$ & $\begin{array}{l}8+ \\
6+ \\
5+\end{array}$ \\
\hline 1 & $\mathbf{B}$ & 1 & $\begin{array}{l}1 \\
2 \\
3 \\
4 \\
4\end{array}$ & $1: 400$ & $\begin{array}{l}0 \\
\pm \\
+ \\
+\end{array}$ & $\begin{array}{l}0 \\
0 \\
0 \\
+\end{array}$ & $\begin{array}{l}0 \\
0 \\
0 \\
\pm\end{array}$ & $\begin{array}{l}+ \\
+ \\
+ \\
+\end{array}$ & $\begin{array}{l}+ \\
+ \\
+\end{array}$ & $\frac{ \pm}{ \pm}$ & $\begin{array}{l}\text { Strongly positive, } \\
\text { Strongly positive, } \\
\text { Strongly positive, } \\
\text { Negative, }\end{array}$ & $\begin{array}{r}10+ \\
6+ \\
5+ \\
-\end{array}$ \\
\hline 1 & B & 2 & $\begin{array}{l}1 \\
2 \\
3 \\
4 \\
4\end{array}$ & $1: 400$ & $\begin{array}{l}0 \\
\operatorname{tr} \\
+ \\
+\end{array}$ & $\begin{array}{l}0 \\
0 \\
\mathbf{t r} \\
+\end{array}$ & $\begin{array}{c}0 \\
0 \\
0 \\
\pm\end{array}$ & $\begin{array}{l}+ \\
+ \\
+ \\
+\end{array}$ & $\begin{array}{l}+ \\
+ \\
+ \\
+\end{array}$ & $\begin{array}{l} \pm \\
\pm \\
\pm \\
\pm\end{array}$ & $\begin{array}{l}\text { Strongly positive, } \\
\text { Strongly positive, } \\
\text { Strongly positive, } \\
\text { Negative, }\end{array}$ & $\begin{array}{r}10+ \\
8+ \\
4+ \\
-\end{array}$ \\
\hline 1 & $\mathrm{~B}$ & 3 & $\begin{array}{l}1 \\
2 \\
3 \\
4 \\
4\end{array}$ & $1: 400$ & $\begin{array}{l}\operatorname{tr} \\
\pm \\
+ \\
+\end{array}$ & $\begin{array}{c}0 \\
0 \\
\text { tr } \\
+\end{array}$ & $\begin{array}{c}0 \\
0 \\
0 \\
\pm\end{array}$ & $\begin{array}{l}+ \\
+ \\
+ \\
+\end{array}$ & $\begin{array}{l}+ \\
+ \\
+ \\
+\end{array}$ & $\begin{array}{l} \pm \\
\pm \\
\pm\end{array}$ & $\begin{array}{l}\text { Strongly positive, } \\
\text { Strongly positive, } \\
\text { Strongly positive, } \\
\text { Negative, }\end{array}$ & $\begin{array}{l}8+ \\
6+ \\
4+ \\
-\end{array}$ \\
\hline 1 & B & 4 & $\begin{array}{l}1 \\
2 \\
3 \\
4\end{array}$ & $1: 400$ & $\begin{array}{l}\mathbf{t r} \\
+ \\
+ \\
+\end{array}$ & $\begin{array}{l}0 \\
\operatorname{tr} \\
\operatorname{tr} \\
+\end{array}$ & $\begin{array}{c}0 \\
0 \\
0 \\
\text { tr }\end{array}$ & $\begin{array}{l}+ \\
+ \\
+ \\
+\end{array}$ & $\begin{array}{l}+ \\
+ \\
+ \\
+\end{array}$ & $\begin{array}{l}\operatorname{tr} \\
\operatorname{tr} \\
\mathbf{t r} \\
\operatorname{tr}\end{array}$ & $\begin{array}{l}\text { Strongly positive, } \\
\text { Strongly positive, } \\
\text { Strongly positive, } \\
\text { Negative, }\end{array}$ & $\begin{array}{l}8+ \\
3+ \\
3+ \\
-\end{array}$ \\
\hline 4 & A & 0 & $\begin{array}{l}1 \\
2 \\
3 \\
4 \\
4\end{array}$ & $1: 400$ & $\begin{array}{l}\operatorname{tr} \\
\pm \\
+ \\
+\end{array}$ & $\begin{array}{r}0 \\
0 \\
0 \\
+\end{array}$ & $\begin{array}{l}0 \\
0 \\
0 \\
\pm\end{array}$ & $\begin{array}{l}+ \\
+ \\
+\end{array}$ & + & $\frac{ \pm}{ \pm}$ & $\begin{array}{l}\text { Strongly positive, } \\
\text { Strongly positive, } \\
\text { Strongly positive, } \\
\text { Negative, }\end{array}$ & $\begin{array}{l}8+ \\
6+ \\
5+\end{array}$ \\
\hline 2 & $A$ & 0 & $\begin{array}{l}5 \\
5 \\
6 \\
7 \\
8\end{array}$ & $1: 400$ & $\begin{array}{l}+ \\
+ \\
+ \\
+\end{array}$ & $\begin{array}{c} \pm \\
\pm \\
+1\end{array}$ & $\begin{array}{l}0 \\
0 \\
0 \\
\pm\end{array}$ & $\begin{array}{l}+ \\
+ \\
+ \\
+\end{array}$ & $\begin{array}{l}+ \\
+ \\
+\end{array}$ & $\frac{ \pm}{ \pm}$ & $\begin{array}{l}\text { Strongly positive, } \\
\text { Strongly positive, } \\
\text { Strongly positive, } \\
\text { Negative, }\end{array}$ & $\begin{array}{l}3+ \\
3+ \\
4+\end{array}$ \\
\hline 2 & B & 1 & $\begin{array}{l}5 \\
6 \\
7 \\
8\end{array}$ & $1: 400$ & $\begin{array}{l}+ \\
+ \\
+ \\
+\end{array}$ & 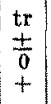 & $\begin{array}{c}0 \\
0 \\
0 \\
\pm\end{array}$ & $\begin{array}{l}+ \\
+ \\
+ \\
+\end{array}$ & $\begin{array}{l}+ \\
+ \\
+ \\
+\end{array}$ & $\begin{array}{l} \pm \\
\pm \\
\pm \\
\pm\end{array}$ & $\begin{array}{l}\text { Strongly positive, } \\
\text { Strongly positive, } \\
\text { Strongly positive, } \\
\text { Negative, }\end{array}$ & $\begin{array}{l}4+ \\
3+ \\
5+ \\
-\end{array}$ \\
\hline 2 & B & 2 & $\begin{array}{l}5 \\
5 \\
6 \\
7 \\
8\end{array}$ & $1: 400$ & $\begin{array}{l}+ \\
+ \\
+ \\
+\end{array}$ & $\frac{ \pm}{ \pm}$ & $\begin{array}{l}0 \\
0 \\
0 \\
\pm\end{array}$ & $\begin{array}{l}+ \\
+ \\
+ \\
+\end{array}$ & $\begin{array}{l}+ \\
+ \\
+\end{array}$ & $\frac{ \pm}{ \pm}$ & $\begin{array}{l}\text { Strongly positive, } \\
\text { Strongly positive, } \\
\text { Strongly positive, } \\
\text { Negative, }\end{array}$ & $\begin{array}{l}3+ \\
3+ \\
4+ \\
-\end{array}$ \\
\hline 2 & B & 3 & $\begin{array}{l}5 \\
6 \\
7 \\
8 \\
8\end{array}$ & $1: 400$ & $\begin{array}{l}+ \\
+ \\
+ \\
+\end{array}$ & $\frac{ \pm}{+1}+$ & $\begin{array}{c}0 \\
0 \\
0 \\
\pm\end{array}$ & $\begin{array}{l}+ \\
+ \\
+ \\
+\end{array}$ & $\begin{array}{l}+ \\
+ \\
+ \\
+\end{array}$ & $\frac{ \pm}{ \pm}$ & $\begin{array}{l}\text { Strongly positive, } \\
\text { Moderately positive, } \\
\text { Strongly positive, } \\
\text { Negative, }\end{array}$ & $\begin{array}{l}3+ \\
3+ \\
3+\end{array}$ \\
\hline 2 & B & 4 & $\begin{array}{l}5 \\
6 \\
7 \\
8\end{array}$ & $1: 400$ & $\begin{array}{c}+ \\
+ \\
+ \\
+\end{array}$ & $\frac{ \pm}{ \pm}$ & $\begin{array}{c}0 \\
0 \\
0 \\
\operatorname{tr}\end{array}$ & $\begin{array}{c}+ \\
+ \\
+ \\
+\end{array}$ & $\begin{array}{c}+ \\
+ \\
+ \\
+\end{array}$ & $\begin{array}{l}\operatorname{tr} \\
\operatorname{tr} \\
\operatorname{tr} \\
\operatorname{tr} \\
\end{array}$ & $\begin{array}{l}\text { Moderately positive, } \\
\text { Moderately positive, } \\
\text { Strongly positive, } \\
\text { Negative, }\end{array}$ & $\begin{array}{l}2+ \\
2+ \\
3+\end{array}$ \\
\hline 4 & $A$ & 0 & $\begin{array}{l}5 \\
6 \\
7 \\
8 \\
8\end{array}$ & $\therefore 400$ & $\begin{array}{c}+ \\
+ \\
+ \\
+\end{array}$ & $\begin{array}{c} \pm \\
\pm \\
t r \\
+\end{array}$ & $\begin{array}{l}0 \\
0 \\
0 \\
\pm\end{array}$ & $\begin{array}{l}+ \\
+ \\
+ \\
+\end{array}$ & $\begin{array}{c}+ \\
+ \\
+\end{array}$ & $\frac{ \pm}{ \pm}$ & $\begin{array}{l}\text { Strongly positive, } \\
\text { Strongly positive, } \\
\text { Strongly positive, } \\
\text { Negative, }\end{array}$ & $\begin{array}{l}3+ \\
3+ \\
4+\end{array}$ \\
\hline 3 & A & 0 & $\begin{array}{r}9 \\
10 \\
11 \\
12\end{array}$ & $1: 400$ & $\begin{array}{l}+ \\
+ \\
+ \\
+\end{array}$ & $\begin{array}{c}\operatorname{tr} \\
0 \\
0 \\
+\end{array}$ & $\begin{array}{l}0 \\
0 \\
0 \\
\pm\end{array}$ & $\begin{array}{l}+ \\
+ \\
+ \\
+\end{array}$ & $\begin{array}{l}+ \\
+ \\
+\end{array}$ & $\frac{ \pm}{ \pm}$ & $\begin{array}{l}\text { Strongly positive, } \\
\text { Strongly positive, } \\
\text { Strongly positive, } \\
\text { Negative, }\end{array}$ & $\begin{array}{l}4+ \\
5+ \\
5+\end{array}$ \\
\hline
\end{tabular}

* Explanation: $0=$ no hemolysis; tr (trace) $=$ hemolysis up to $50 \% ; \pm=$ hemolysis bet ween $50 \%$ and $100 \% ;+=$ complete hemolysis. 
Frozen Complement in Wassermann Reaction

TABLE 1-Continued

Mixed Frozen Complement Tested at Intervals of a Week

\begin{tabular}{|c|c|c|c|c|c|c|c|c|c|c|c|c|}
\hline \multirow{3}{*}{$\begin{array}{l}\text { Number } \\
\text { of } \\
\text { Comple- } \\
\text { ment }\end{array}$} & \multirow{3}{*}{$\begin{array}{c}\text { Portions } \\
\text { A-Fresh } \\
\text { B-Frozen }\end{array}$} & \multirow{3}{*}{$\begin{array}{c}\text { Age } \\
\text { Weeks }\end{array}$} & \multirow{3}{*}{$\begin{array}{c}\text { Number } \\
\text { of } \\
\text { Serum }\end{array}$} & \multirow{3}{*}{$\begin{array}{l}\text { Dilu- } \\
\text { tion } \\
\text { of } \\
\text { Ambo- } \\
\text { ceptor }\end{array}$} & \multicolumn{6}{|c|}{ Readings } & \multirow{3}{*}{\multicolumn{2}{|c|}{ Aesults }} \\
\hline & & & & & \multicolumn{3}{|c|}{$\begin{array}{c}\text { Antigen } \\
\text { Tubes }\end{array}$} & \multicolumn{3}{|c|}{$\begin{array}{c}\text { Control } \\
\text { Tubes }\end{array}$} & & \\
\hline & & & & & 1 & 2 & 3 & $1^{\prime}$ & $2^{\prime}$ & $3^{\prime}$ & & \\
\hline 3 & $\mathbf{B}$ & 1 & $\begin{array}{r}9 \\
10 \\
11 \\
12\end{array}$ & $1: 400$ & $\begin{array}{l} \pm \\
\pm \\
\pm \\
+\end{array}$ & $\begin{array}{c}0 \\
0 \\
0 \\
+\end{array}$ & $\begin{array}{r}0 \\
0 \\
0 \\
\pm\end{array}$ & $\begin{array}{l}+ \\
+ \\
+ \\
+\end{array}$ & $\begin{array}{l}+ \\
+ \\
+ \\
+\end{array}$ & $\begin{array}{l} \pm \\
\pm \\
\pm\end{array}$ & $\begin{array}{l}\text { Strongly positive, } \\
\text { Strongly positive, } \\
\text { Strongly positive, } \\
\text { Negative, }\end{array}$ & $\begin{array}{l}5+ \\
6+- \\
6+ \\
-\end{array}$ \\
\hline 3 & B & 2 & $\begin{array}{r}9 \\
10 \\
11 \\
12\end{array}$ & $1: 400$ & $\begin{array}{l}+ \\
\operatorname{tr} \\
\operatorname{tr} \\
+\end{array}$ & $\begin{array}{l}0 \\
0 \\
0 \\
+\end{array}$ & $\begin{array}{l}0 \\
0 \\
0 \\
\pm\end{array}$ & $\begin{array}{l}+ \\
+ \\
+ \\
+\end{array}$ & $\begin{array}{l}+ \\
+ \\
+ \\
+\end{array}$ & $\begin{array}{l}\operatorname{tr} \\
\operatorname{tr} \\
\operatorname{tr} \\
\pm\end{array}$ & $\begin{array}{l}\text { Strongly positive, } \\
\text { Strongly positive, } \\
\text { Strongly positive, } \\
\text { Negative, }\end{array}$ & $\begin{array}{l}4+ \\
6+ \\
6+ \\
-\end{array}$ \\
\hline 3 & B & 3 & $\begin{array}{r}9 \\
10 \\
11 \\
12\end{array}$ & $1: 400$ & $\begin{array}{l}+ \\
+ \\
+ \\
+\end{array}$ & $\begin{array}{c:}\operatorname{tr} \\
0 \\
0 \\
+\end{array}$ & $\begin{array}{c}0 \\
0 \\
0 \\
\text { tr }\end{array}$ & $\begin{array}{l}+ \\
+ \\
+ \\
+\end{array}$ & $\begin{array}{l}+ \\
+ \\
+ \\
+\end{array}$ & $\begin{array}{l}\operatorname{tr} \\
\operatorname{tr} \\
\operatorname{tr} \\
\operatorname{tr}\end{array}$ & $\begin{array}{l}\text { Strongly positive, } \\
\text { Strongly positive, } \\
\text { Strongly positive, } \\
\text { Negative, }\end{array}$ & $\begin{array}{l}3+ \\
4+ \\
5+ \\
-\end{array}$ \\
\hline 3 & $\mathbf{B}$ & 4 & $\begin{array}{r}9 \\
10 \\
11 \\
12\end{array}$ & $1: 400$ & $\begin{array}{l}+ \\
\pm \\
\pm \\
+\end{array}$ & $\begin{array}{l:l}0 & \\
0 & \\
0 & \\
+ & \end{array}$ & $\begin{array}{c}0 \\
0 \\
0 \\
\operatorname{tr}\end{array}$ & $\begin{array}{l}+ \\
+ \\
+ \\
+\end{array}$ & $\begin{array}{l}+ \\
+ \\
+ \\
+\end{array}$ & $\begin{array}{c}0 \\
0 \\
0 \\
\operatorname{tr}\end{array}$ & $\begin{array}{l}\text { Strongly positive, } \\
\text { Strongly positive, } \\
\text { Strongly positive, } \\
\text { Negative, }\end{array}$ & $\begin{array}{l}3+ \\
4+ \\
4+ \\
-\end{array}$ \\
\hline 4 & A & 0 & $\begin{array}{r}9 \\
10 \\
11 \\
12\end{array}$ & $1: 400$ & $\begin{array}{l}+ \\
\pm \\
+ \\
+\end{array}$ & $\begin{array}{c}\mathbf{t r} \\
0 \\
0 \\
+\end{array}$ & $\begin{array}{l}0 \\
0 \\
0 \\
\pm\end{array}$ & $\begin{array}{l}+ \\
+ \\
+ \\
+\end{array}$ & $\begin{array}{l}+ \\
+ \\
+ \\
+\end{array}$ & $\begin{array}{l} \pm \\
\pm \\
\pm \\
\pm\end{array}$ & $\begin{array}{l}\text { Strongly positive, } \\
\text { Strongly positive, } \\
\text { Strongly positive, } \\
\text { Negative, }\end{array}$ & $\begin{array}{l}4+ \\
6+ \\
5+ \\
-\end{array}$ \\
\hline 5 & A & 0 & $\begin{array}{l}13 \\
14 \\
15 \\
16\end{array}$ & $1: 400$ & $\begin{array}{l}+ \\
+ \\
+ \\
+\end{array}$ & $\begin{array}{l}\frac{ \pm}{\operatorname{tr}} \\
\operatorname{tr} \\
+\end{array}$ & $\begin{array}{l}0 \\
0 \\
0 \\
\pm\end{array}$ & $\begin{array}{l}+ \\
+ \\
+ \\
+\end{array}$ & $\begin{array}{l}+ \\
+ \\
+ \\
+ \\
+\end{array}$ & $\frac{ \pm}{ \pm}$ & $\begin{array}{l}\text { Strongly positive, } \\
\text { Strongly positive, } \\
\text { Strongly positive, } \\
\text { Negative, }\end{array}$ & $\begin{array}{l}3+ \\
4+ \\
4+ \\
-\end{array}$ \\
\hline 5 & B & 1 & $\begin{array}{l}13 \\
14 \\
15 \\
16\end{array}$ & $1: 400$ & $\begin{array}{l}+ \\
+ \\
+ \\
+\end{array}$ & $\begin{array}{c}\operatorname{tr} \\
0 \\
0 \\
+\end{array}$ & $\begin{array}{l}0 \\
0 \\
0 \\
\pm\end{array}$ & $\begin{array}{l}+ \\
+ \\
+ \\
+\end{array}$ & $\begin{array}{l}+ \\
+ \\
+ \\
+\end{array}$ & $\begin{array}{l}\frac{ \pm}{ \pm} \\
\frac{ \pm}{ \pm}\end{array}$ & $\begin{array}{l}\text { Strongly positive, } \\
\text { Strongly positive, } \\
\text { Strongly positive, } \\
\text { Negative, }\end{array}$ & $\begin{array}{l}4+ \\
5+ \\
5+ \\
-\end{array}$ \\
\hline 5 & B & $\therefore$ & $\begin{array}{l}13 \\
14 \\
15 \\
16\end{array}$ & $1: 400$ & $\begin{array}{l}+ \\
+ \\
+ \\
+\end{array}$ & $\begin{array}{c}\operatorname{tr} \\
0 \\
0 \\
+\end{array}$ & $\begin{array}{c}0 \\
0 \\
0 \\
\text { tr }\end{array}$ & $\begin{array}{l}+ \\
+ \\
+ \\
+\end{array}$ & $\begin{array}{l}+1 \\
+1 \\
+1 \\
+\end{array}$ & $\begin{array}{l}\operatorname{tr} \\
\operatorname{tr} \\
\operatorname{tr} \\
\operatorname{tr}\end{array}$ & $\begin{array}{l}\text { Strongly positive, } \\
\text { Strongly positive, } \\
\text { Strongly positive, } \\
\text { Negative, }\end{array}$ & $\begin{array}{l}3+ \\
4+ \\
4+ \\
-\end{array}$ \\
\hline 5 & B & 3 & $\begin{array}{l}13 \\
14 \\
15 \\
16\end{array}$ & $1: 400$ & $\begin{array}{c}\text { tr } \\
0 \\
0 \\
+\end{array}$ & $\begin{array}{c}0 \\
0 \\
0 \\
\mathrm{tr}\end{array}$ & $\begin{array}{l}0 \\
0 \\
0 \\
0\end{array}$ & $\begin{array}{l}+ \\
+ \\
+ \\
+\end{array}$ & $\begin{array}{l} \pm \\
\operatorname{tr} \\
\operatorname{tr} \\
\operatorname{tr}\end{array}$ & $\begin{array}{l}0 \\
0 \\
0 \\
0\end{array}$ & $\begin{array}{l}\text { Strongly positive, } \\
\text { Strongly positive, } \\
\text { Strongly positive, } \\
\text { Negative, }\end{array}$ & $\begin{array}{l}4+ \\
4+ \\
4+ \\
-\end{array}$ \\
\hline 5 & B & 4 & $\begin{array}{l}13 \\
14 \\
15 \\
16\end{array}$ & $1: 400$ & $\begin{array}{l} \pm \\
\frac{ \pm}{\operatorname{tr}} \\
\operatorname{tr} \\
+\end{array}$ & $\begin{array}{c}0 \\
0 \\
0 \\
\operatorname{tr}\end{array}$ & $\begin{array}{l}0 \\
0 \\
0 \\
0\end{array}$ & $\begin{array}{l} \pm \\
\pm \\
\pm \\
\pm\end{array}$ & $\begin{array}{c:}\operatorname{tr} \\
0 \\
\operatorname{tr} \\
\operatorname{tr}\end{array}$ & $\begin{array}{l}0 \\
0 \\
0 \\
0\end{array}$ & $\begin{array}{l}\text { Moderately positive, } \\
\text { Weakly positive, } \\
\text { Unft } \\
\text { Unflt }\end{array}$ & $\begin{array}{l}2+ \\
1+\end{array}$ \\
\hline 8 & A & 0 & $\begin{array}{l}12 \\
14 \\
15 \\
16\end{array}$ & $1: 400$ & $\begin{array}{l}+ \\
+ \\
\pm \\
+\end{array}$ & $\begin{array}{c}\operatorname{tr} \\
0 \\
0 \\
+\end{array}$ & $\begin{array}{c}0 \\
0 \\
0 \\
\pm\end{array}$ & $\begin{array}{l}+ \\
+ \\
+ \\
+\end{array}$ & $\begin{array}{l}+ \\
+ \\
+ \\
+\end{array}$ & $\frac{ \pm}{ \pm}$ & $\begin{array}{l}\text { Strongly positive, } \\
\text { Strongly positjve, } \\
\text { Strongly positive, } \\
\text { Negative, }\end{array}$ & $\begin{array}{l}4+ \\
5+ \\
6+ \\
-\end{array}$ \\
\hline f: & $A$ & 0 & $\begin{array}{l}17 \\
18 \\
19 \\
20\end{array}$ & $1: 400$ & $\begin{array}{l}\operatorname{tr} \\
\pm \\
\operatorname{tr} \\
+\end{array}$ & $\begin{array}{l}0 \\
0 \\
0 \\
+\end{array}$ & $\begin{array}{c}0 \\
0 \\
0 \\
\pm\end{array}$ & $\begin{array}{l}+ \\
+ \\
+ \\
+\end{array}$ & $\begin{array}{l}+ \\
+ \\
+ \\
+\end{array}$ & $\begin{array}{l} \pm \\
\pm \\
\pm \\
\pm\end{array}$ & $\begin{array}{l}\text { Strongly positive, } \\
\text { Strongly positive, } \\
\text { Strongly positive, } \\
\text { Negative, }\end{array}$ & $\begin{array}{l}8+ \\
6+ \\
8+ \\
-\end{array}$ \\
\hline 6 & B & 1 & $\begin{array}{l}17 \\
18 \\
19 \\
20\end{array}$ & $1: 400$ & $\begin{array}{c}0 \\
\operatorname{tr} \\
0 \\
+\end{array}$ & $\begin{array}{l}0 \\
0 \\
0 \\
+\end{array}$ & $\begin{array}{c}0 \\
0 \\
0 \\
\pm\end{array}$ & $\begin{array}{l}+ \\
+ \\
+ \\
+\end{array}$ & $\begin{array}{l}+ \\
+ \\
+ \\
+\end{array}$ & $\begin{array}{l}\frac{ \pm}{ \pm} \\
\frac{ \pm}{ \pm}\end{array}$ & $\begin{array}{l}\text { Strongly positive, } \\
\text { Strongly positive, } \\
\text { Strongly positive, } \\
\text { Negative, }\end{array}$ & $\begin{array}{r}10+ \\
8+ \\
10+ \\
-\end{array}$ \\
\hline
\end{tabular}


TABLE 1-Continued

Mixed Frozen Complement Tested at Intervals of a Weex

\begin{tabular}{|c|c|c|c|c|c|c|c|c|c|c|c|c|}
\hline \multirow{3}{*}{$\begin{array}{l}\text { Number } \\
\text { of } \\
\text { Comple } \\
\text { ment }\end{array}$} & \multirow{3}{*}{$\begin{array}{c}\text { Portions } \\
\text { A-Fresh } \\
\text { B-Frozen }\end{array}$} & \multirow{3}{*}{$\begin{array}{c}\text { Age, } \\
\text { Weeks }\end{array}$} & \multirow{3}{*}{$\begin{array}{c}\text { Number } \\
\text { of } \\
\text { Serum }\end{array}$} & \multirow{3}{*}{$\begin{array}{l}\text { Dilu- } \\
\text { tion } \\
\text { of } \\
\text { Ambo- } \\
\text { ceptor }\end{array}$} & \multicolumn{6}{|c|}{ Readings } & \multirow{3}{*}{\multicolumn{2}{|c|}{ Aesults }} \\
\hline & & & & & \multicolumn{3}{|c|}{$\begin{array}{c}\text { Antigen } \\
\text { Tubes }\end{array}$} & \multicolumn{3}{|c|}{$\begin{array}{l}\text { Control } \\
\text { Tubes }\end{array}$} & & \\
\hline & & & & & 1 & 2 & 3 & $1^{\prime}$ & $2^{\prime}$ & $3^{\prime}$ & & \\
\hline 6 & $\mathbf{B}$ & 2 & $\begin{array}{l}17 \\
18 \\
19 \\
20\end{array}$ & $1: 400$ & $\begin{array}{l}0 \\
0 \\
0 \\
+\end{array}$ & $\begin{array}{l}0 \\
0 \\
0 \\
+\end{array}$ & $\begin{array}{l}0 \\
0 \\
0 \\
0\end{array}$ & $\begin{array}{l}+ \\
+ \\
+ \\
+\end{array}$ & $\begin{array}{l}+ \\
+ \\
+ \\
+\end{array}$ & $\begin{array}{l}0 \\
0 \\
0 \\
0\end{array}$ & $\begin{array}{l}\text { Strongly positive, } \\
\text { Strongly positive, } \\
\text { Strongly positive, } \\
\text { Negative, }\end{array}$ & $\begin{array}{l}6+ \\
6+ \\
6+ \\
-\end{array}$ \\
\hline 6 & B & 3 & $\begin{array}{l}17 \\
18 \\
19 \\
20\end{array}$ & $1: 400$ & $\begin{array}{l}0 \\
0 \\
0 \\
+\end{array}$ & $\begin{array}{c}0 \\
0 \\
0 \\
\operatorname{tr}\end{array}$ & $\begin{array}{l}0 \\
0 \\
0 \\
0\end{array}$ & 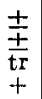 & $\begin{array}{l}\operatorname{tr} \\
\operatorname{tr} \\
\operatorname{tr} \\
\operatorname{tr}\end{array}$ & $\begin{array}{l}0 \\
0 \\
0 \\
0\end{array}$ & $\begin{array}{l}\text { Unflt } \\
\text { Unflt } \\
\text { Unftt } \\
\text { Negative, }\end{array}$ & - \\
\hline 6 & B & 4 & $\begin{array}{l}17 \\
18 \\
19 \\
20\end{array}$ & $1: 400$ & $\begin{array}{c}0 \\
0 \\
0 \\
\pm\end{array}$ & $\begin{array}{c}0 \\
0 \\
0 \\
\operatorname{tr}\end{array}$ & $\begin{array}{l}0 \\
0 \\
0 \\
0\end{array}$ & $\begin{array}{l}\operatorname{tr} \\
\operatorname{tr} \\
\operatorname{tr} \\
\pm\end{array}$ & $\begin{array}{l}\operatorname{tr} \\
\operatorname{tr} \\
\operatorname{tr} \\
\operatorname{tr}\end{array}$ & $\begin{array}{l}0 \\
0 \\
0 \\
0\end{array}$ & $\begin{array}{l}\text { Unfit } \\
\text { Unfit } \\
\text { Unfit } \\
\text { Unfit }\end{array}$ & \\
\hline 8 & $\mathbf{A}$ & 0 & $\begin{array}{l}17 \\
18 \\
19 \\
20\end{array}$ & $1: 400$ & $\begin{array}{c}0 \\
\operatorname{tr} \\
0 \\
+\end{array}$ & $\begin{array}{l}0 \\
0 \\
0 \\
+\end{array}$ & $\begin{array}{l}0 \\
0 \\
0 \\
\pm\end{array}$ & $\begin{array}{l}+ \\
+ \\
+ \\
+\end{array}$ & $\begin{array}{l}+ \\
+ \\
+ \\
+\end{array}$ & $\begin{array}{l} \pm \\
\pm \\
\pm \\
\pm\end{array}$ & $\begin{array}{l}\text { Strongly positive, } \\
\text { Strongly positive, } \\
\text { Strongly positive, } \\
\text { Negative, }\end{array}$ & $\begin{array}{r}10+ \\
8+ \\
10+ \\
-\end{array}$ \\
\hline 7 & $\mathbf{A}$ & 0 & $\begin{array}{l}21 \\
22 \\
23 \\
24\end{array}$ & $1: 400$ & $\begin{array}{l}+ \\
+ \\
+ \\
+\end{array}$ & $\begin{array}{l}\operatorname{tr} \\
\operatorname{tr} \\
\pm \\
\pm\end{array}$ & $\begin{array}{r}0 \\
0 \\
0 \\
\pm\end{array}$ & $\begin{array}{l}+ \\
+ \\
+ \\
+\end{array}$ & $\begin{array}{l}+ \\
+ \\
+ \\
+\end{array}$ & $\begin{array}{l} \pm \\
\pm \\
\pm \\
\pm\end{array}$ & $\begin{array}{l}\text { Strongly positive, } \\
\text { Strongly positive, } \\
\text { Strongly positive, } \\
\text { Negative, }\end{array}$ & $\begin{array}{l}4+ \\
4+ \\
3+ \\
-\end{array}$ \\
\hline 7 & B & 1 & $\begin{array}{l}21 \\
22 \\
23 \\
24\end{array}$ & $1: 400$ & $\begin{array}{l}+ \\
+ \\
+ \\
+\end{array}$ & \begin{tabular}{c|c}
0 \\
0 \\
$\operatorname{tr}$ \\
+
\end{tabular} & $\begin{array}{r}0 \\
0 \\
0 \\
\pm\end{array}$ & $\begin{array}{l}+ \\
+ \\
+ \\
+\end{array}$ & $\begin{array}{l}+ \\
+ \\
+ \\
+\end{array}$ & $\begin{array}{l} \pm \\
\pm \\
\pm\end{array}$ & $\begin{array}{l}\text { Strongly positive, } \\
\text { Strongly positive, } \\
\text { Strongly positive, } \\
\text { Negative, }\end{array}$ & $\begin{array}{l}5+ \\
5+ \\
4+ \\
+\end{array}$ \\
\hline$i$ & $\mathbf{B}$ & 2 & $\begin{array}{l}21 \\
22 \\
28 \\
24\end{array}$ & $1: 400$ & $\begin{array}{l}\operatorname{tr} \\
\pm \\
+ \\
+\end{array}$ & $\begin{array}{l}0 \\
0 \\
0 \\
+\end{array}$ & $\begin{array}{l}0 \\
0 \\
0 \\
0\end{array}$ & $\begin{array}{l}+ \\
+ \\
+ \\
+\end{array}$ & $\begin{array}{l}+ \\
+ \\
+ \\
+\end{array}$ & $\begin{array}{l}0 \\
0 \\
0 \\
0\end{array}$ & $\begin{array}{l}\text { Strongly positive, } \\
\text { Strongly positive, } \\
\text { Strongly positive, } \\
\text { Negative, }\end{array}$ & $\begin{array}{r}5+ \\
4+ \\
3+ \\
-\end{array}$ \\
\hline 7 & B & 3 & $\begin{array}{l}21 \\
22 \\
23 \\
24\end{array}$ & $1: 400$ & $\begin{array}{l}+ \\
+ \\
+ \\
+\end{array}$ & $\begin{array}{l}0 \\
0 \\
0 \\
\pm\end{array}$ & $\begin{array}{l}0 \\
0 \\
0 \\
0\end{array}$ & $\begin{array}{l}+ \\
+ \\
+ \\
+\end{array}$ & $\begin{array}{l} \pm \\
\pm \\
\pm \\
\pm\end{array}$ & $\begin{array}{l}0 \\
0 \\
0 \\
0\end{array}$ & $\begin{array}{l}\text { Moderately positive, } \\
\text { Moderately positive, } \\
\text { Moderately positive, } \\
\text { Negative, }\end{array}$ & $\begin{array}{l}2+ \\
2+ \\
2+ \\
+\end{array}$ \\
\hline 7 & B & 4 & $\begin{array}{l}21 \\
22 \\
23 \\
24\end{array}$ & $1: 400$ & $\begin{array}{l}\frac{ \pm}{ \pm} \\
\frac{1}{+} \\
+\end{array}$ & $\begin{array}{c}0 \\
0 \\
0 \\
t r\end{array}$ & $\begin{array}{l}0 \\
0 \\
0 \\
0\end{array}$ & $\begin{array}{l}+ \\
+ \\
+ \\
+\end{array}$ & $\begin{array}{l}\mathrm{tr} \\
\pm \\
\pm \\
\mathrm{tr}\end{array}$ & $\begin{array}{l}0 \\
0 \\
0 \\
0\end{array}$ & $\begin{array}{l}\text { Moderately positive, } \\
\text { Strongly positive, } \\
\text { Strongly positive, } \\
\text { Negative, }\end{array}$ & $\begin{array}{l}2+ \\
3+ \\
3+ \\
-\end{array}$ \\
\hline 8 & $\mathbf{A}$ & 0 & $\begin{array}{l}21 \\
22 \\
23 \\
24\end{array}$ & $1: 400$ & $\begin{array}{l}+ \\
+ \\
+ \\
+\end{array}$ & $\begin{array}{l}0 \\
\operatorname{tr} \\
\operatorname{tr} \\
+\end{array}$ & $\begin{array}{l}0 \\
0 \\
0 \\
\pm\end{array}$ & $\begin{array}{l}+ \\
+ \\
+ \\
+\end{array}$ & $\begin{array}{l}+ \\
+ \\
+ \\
+\end{array}$ & $\begin{array}{l} \pm \\
\pm \\
\pm \\
\pm\end{array}$ & $\begin{array}{l}\text { Strongly positive, } \\
\text { Strongly positive, } \\
\text { Strongly positive, } \\
\text { Negative, }\end{array}$ & $\begin{array}{l}5+ \\
4+ \\
4+ \\
-\end{array}$ \\
\hline
\end{tabular}

serums while fresh and portion $B$ of each number was frozen, was kept frozen and was tested on the same human serums at intervals of a week for 4 weeks. At the end of 4 weeks the human serums were tested with fresh control complements (Nos. 12 and 16) in order to detect any changes in the human serums.

The results obtained with unmixed guinea-pig complements are shown in table 2. Frozen complement one week old frequently gave stronger positive results than did the same complement while fresh. Complement that had been kept frozen for 2 weeks gave results that 
Frozen Complement in Wassermann Reaction

TABLE

Unmixed Frozen Complement Tested at Intervals of a Week

\begin{tabular}{|c|c|c|c|c|c|c|c|c|c|c|c|}
\hline \multirow{3}{*}{$\begin{array}{l}\text { Number } \\
\text { of } \\
\text { Comple- } \\
\text { ment }\end{array}$} & \multirow{3}{*}{$\begin{array}{c}\text { Portions } \\
\text { A-Fresh } \\
\text { B-Frozen }\end{array}$} & \multirow{3}{*}{$\begin{array}{l}\text { Age, } \\
\text { Weeks }\end{array}$} & \multirow{3}{*}{$\begin{array}{c}\text { Number } \\
\text { of } \\
\text { Serum }\end{array}$} & \multirow{3}{*}{$\begin{array}{l}\text { Dilu- } \\
\text { tion } \\
\text { of } \\
\text { Ambo- } \\
\text { ceptor }\end{array}$} & \multicolumn{6}{|c|}{ Readings* } & \multirow{3}{*}{ Results } \\
\hline & & & & & \multicolumn{3}{|c|}{$\begin{array}{c}\text { Antigen } \\
\text { Tubes }\end{array}$} & \multicolumn{3}{|c|}{$\begin{array}{l}\text { Control } \\
\text { Tubes }\end{array}$} & \\
\hline & & & & & 1 & 2 & 3 & $1^{\prime}$ & $z^{\prime}$ & $3^{\prime}$ & \\
\hline 9 & $A$ & 0 & $\begin{array}{l}25 \\
26 \\
27 \\
28\end{array}$ & $1: 400$ & $\begin{array}{l}+ \\
+ \\
+ \\
+\end{array}$ & $\begin{array}{l} \pm \\
\operatorname{tr} \\
\operatorname{tr} \\
+\end{array}$ & $\begin{array}{c}0 \\
0 \\
0 \\
\mathrm{tr}\end{array}$ & $\begin{array}{l}+ \\
+ \\
+ \\
+\end{array}$ & $\begin{array}{l}+ \\
+ \\
+ \\
+\end{array}$ & $\begin{array}{l}\operatorname{tr} \\
\operatorname{tr} \\
\operatorname{tr} \\
\operatorname{tr}\end{array}$ & $\begin{array}{ll}\text { Moderately positive, } & 2+ \\
\text { Strongly positive, } & 3+ \\
\text { Strongly positive, } & 3+ \\
\text { Negative, } & -\end{array}$ \\
\hline y & $\mathbf{B}$ & 1 & $\begin{array}{l}25 \\
26 \\
27 \\
28\end{array}$ & $1: 400$ & $\begin{array}{l}+ \\
+ \\
+ \\
+\end{array}$ & $\begin{array}{l}\operatorname{tr} \\
\operatorname{tr} \\
\operatorname{tr} \\
+\end{array}$ & $\begin{array}{c}0 \\
0 \\
0 \\
\mathrm{tr}\end{array}$ & $\begin{array}{l}+ \\
+ \\
+ \\
+\end{array}$ & $\begin{array}{l}+ \\
+ \\
+ \\
+\end{array}$ & $\begin{array}{l}0 \\
\operatorname{tr} \\
\operatorname{tr} \\
\operatorname{tr}\end{array}$ & $\begin{array}{ll}\text { Moderately positive, } & 2+ \\
\text { Strongly positive; } & 3+ \\
\text { Strongly positive, } & 3+ \\
\text { Negative, } & -\end{array}$ \\
\hline$y$ & B & 2 & $\begin{array}{l}25 \\
26 \\
27 \\
29\end{array}$ & $1: 400$ & $\begin{array}{l}+ \\
+ \\
+ \\
+\end{array}$ & $\begin{array}{l}\operatorname{tr} \\
\operatorname{tr} \\
\pm \\
\pm\end{array}$ & $\begin{array}{c}0 \\
0 \\
0 \\
\mathrm{tr}\end{array}$ & $\begin{array}{l}+ \\
+ \\
+ \\
+ \\
+\end{array}$ & $\begin{array}{l}+ \\
+ \\
+ \\
+\end{array}$ & $\begin{array}{l}0 \\
\operatorname{tr} \\
\operatorname{tr} \\
\operatorname{tr}\end{array}$ & $\begin{array}{ll}\text { Moderately positive. } 2+ \\
\text { Strongly positive, } & 3+ \\
\text { Moderately positive, } 2+ \\
\text { Negative, }\end{array}$ \\
\hline 9 & B & 3 & $\begin{array}{l}25 \\
26 \\
27 \\
28\end{array}$ & $1: 400$ & $\begin{array}{l}+ \\
+ \\
+ \\
+\end{array}$ & $\begin{array}{l}\operatorname{tr} \\
\operatorname{tr} \\
\operatorname{tr} \\
+\end{array}$ & $\begin{array}{l}0 \\
0 \\
0 \\
0\end{array}$ & $\begin{array}{l}+ \\
+ \\
+ \\
+\end{array}$ & $\begin{array}{l} \pm \\
+ \\
+ \\
+\end{array}$ & $\begin{array}{l}0 \\
0 \\
0 \\
0\end{array}$ & $\begin{array}{ll}\text { Weakly positive, } & 1+ \\
\text { Moderately positive, } 2+ \\
\text { Moderately positive, } 2+ \\
\text { Negative, }\end{array}$ \\
\hline 9 & B & 4 & $\begin{array}{l}25 \\
26 \\
27 \\
28\end{array}$ & $1: 400$ & $\begin{array}{l}+ \\
+ \\
+ \\
+\end{array}$ & $\begin{array}{l}\operatorname{tr} \\
\operatorname{tr} \\
\operatorname{tr} \\
+\end{array}$ & $\begin{array}{l}0 \\
0 \\
0 \\
0\end{array}$ & $\begin{array}{l}+ \\
+ \\
+ \\
+\end{array}$ & $\begin{array}{l} \pm \\
+ \\
+ \\
+\end{array}$ & $\begin{array}{l}0 \\
0 \\
0 \\
0\end{array}$ & $\begin{array}{ll}\text { Weakly positive, } & 1+ \\
\text { Moderately positive, } & 2+ \\
\text { Moderately positive, } 2+ \\
\text { Negative, }\end{array}$ \\
\hline 12 & $A$ & 0 & $\begin{array}{l}25 \\
26 \\
27 \\
28\end{array}$ & $1: 400$ & $\begin{array}{l}+ \\
+ \\
+ \\
+\end{array}$ & $\begin{array}{l}+ \\
t r \\
\pm \\
+\end{array}$ & $\begin{array}{l}0 \\
0 \\
0 \\
\pm\end{array}$ & $\begin{array}{l}+ \\
+ \\
+ \\
+\end{array}$ & $\begin{array}{l}+ \\
+ \\
+ \\
+\end{array}$ & $\begin{array}{l} \pm \\
\pm \\
\pm\end{array}$ & $\begin{array}{ll}\text { Moderately positive, } & 2+ \\
\text { Strongly positive, } & 4+ \\
\text { Strongly positive, } & 3+ \\
\text { Negative, } & -\end{array}$ \\
\hline 10 & $A$ & 0 & $\begin{array}{l}29 \\
30 \\
31 \\
32\end{array}$ & $1: 400$ & $\begin{array}{l}+ \\
+ \\
+ \\
+\end{array}$ & $\begin{array}{l} \pm \frac{ \pm}{\operatorname{tr}} \\
\pm \\
+\end{array}$ & $\begin{array}{r}0 \\
0 \\
0 \\
\pm\end{array}$ & $\begin{array}{l}+ \\
+ \\
+ \\
+\end{array}$ & $\begin{array}{l}+ \\
+ \\
+ \\
+\end{array}$ & $\begin{array}{l}\operatorname{tr} \\
\pm \\
\pm \\
\pm\end{array}$ & $\begin{array}{ll}\text { Moderately positive, } 2+ \\
\text { Strongly positive, } \\
\text { Strongly positive, } \\
\text { Negative, }\end{array}$ \\
\hline 10 & $B$ & 1 & $\begin{array}{l}29 \\
30 \\
31 \\
32\end{array}$ & $1: 400$ & $\begin{array}{l}+ \\
+ \\
+ \\
+\end{array}$ & $\begin{array}{l}\operatorname{tr} \\
\operatorname{tr} \\
\operatorname{tr} \\
+\end{array}$ & $\begin{array}{r}0 \\
0 \\
0 \\
\pm\end{array}$ & $\begin{array}{l}+ \\
+ \\
+ \\
+\end{array}$ & $\begin{array}{l}+ \\
+ \\
+ \\
+\end{array}$ & $\begin{array}{l}\text { tr } \\
\pm \\
\pm \\
\pm\end{array}$ & $\begin{array}{ll}\text { Strongly positive, } & 3+ \\
\text { Strongly positive, } & 4+ \\
\text { Strongly positive, } & 4+ \\
\text { Negative, } & -\end{array}$ \\
\hline 10 & $\mathbf{B}$ & 2 & $\begin{array}{l}29 \\
30 \\
31 \\
32\end{array}$ & $1: 400$ & $\begin{array}{l}+ \\
+ \\
+ \\
+\end{array}$ & $\begin{array}{l} \pm \\
\operatorname{tr} \\
\pm \\
+\end{array}$ & $\begin{array}{l}0 \\
0 \\
0 \\
\pm\end{array}$ & $\begin{array}{l}+ \\
+ \\
+ \\
+\end{array}$ & $\begin{array}{l}+ \\
+ \\
+ \\
+\end{array}$ & $\begin{array}{l}\text { tr } \\
\pm \\
\pm \\
\pm\end{array}$ & $\begin{array}{ll}\text { Moderately positive, } & 2+ \\
\text { Strongly positive, } & 4+ \\
\text { Strongly positive, } & 3+ \\
\text { Negative, }\end{array}$ \\
\hline 10 & $\mathrm{~B}$ & 3 & $\begin{array}{l}29 \\
30 \\
31 \\
32\end{array}$ & $1: 400$ & $\begin{array}{l}+ \\
+ \\
+ \\
+\end{array}$ & $\begin{array}{l}\operatorname{tr} \\
\operatorname{tr} \\
\operatorname{tr} \\
+\end{array}$ & $\begin{array}{c}0 \\
0 \\
0 \\
\mathrm{tr}\end{array}$ & $\begin{array}{l}+ \\
+ \\
+ \\
+\end{array}$ & $\begin{array}{l}+ \\
+ \\
+ \\
+\end{array}$ & $\begin{array}{l}0 \\
\operatorname{tr} \\
\operatorname{tr} \\
\operatorname{tr}\end{array}$ & $\begin{array}{ll}\text { Moderately positive, } & 2+ \\
\text { Strongly positive, } & 3+ \\
\text { Strongly positive, } & 3+ \\
\text { Negative, } & -\end{array}$ \\
\hline 10 & $B$ & 4 & $\begin{array}{l}29 \\
30 \\
31 \\
32\end{array}$ & $1: 400$ & $\begin{array}{l}+ \\
+ \\
+ \\
+\end{array}$ & $\begin{array}{l}\operatorname{tr} \\
\operatorname{tr} \\
\operatorname{tr} \\
+\end{array}$ & $\begin{array}{c}0 \\
0 \\
0 \\
\operatorname{tr}\end{array}$ & $\begin{array}{l}+ \\
+ \\
+ \\
+\end{array}$ & $\begin{array}{l} \pm \\
+ \\
+ \\
+\end{array}$ & $\begin{array}{c}0 \\
0 \\
0 \\
\mathbf{t r}\end{array}$ & $\begin{array}{ll}\text { Weakly positive, } & 1+ \\
\text { Moderately positive, } 2+ \\
\text { Moderately positive, } 2+ \\
\text { Negative, }\end{array}$ \\
\hline 12 & $\Lambda$ & 0 & $\begin{array}{l}29 \\
30 \\
31 \\
32\end{array}$ & $1: 400$ & $\begin{array}{l}+ \\
+ \\
+ \\
+\end{array}$ & $\begin{array}{l} \pm \\
\operatorname{tr} \\
\frac{ \pm}{+}\end{array}$ & $\begin{array}{l}0 \\
0 \\
0 \\
\pm\end{array}$ & $\begin{array}{l}+ \\
+ \\
+ \\
+\end{array}$ & $\begin{array}{l}+ \\
+ \\
+ \\
+\end{array}$ & $\begin{array}{l} \pm \\
\frac{ \pm}{ \pm} \\
\pm\end{array}$ & $\begin{array}{ll}\text { Strongly positive, } & 3+ \\
\text { Strongly positive, } & 4+ \\
\text { Strongly positive, } & 3+ \\
\text { Negative, } & -\end{array}$ \\
\hline 11 & $\mathbf{A}$ & $\vartheta$ & $\begin{array}{l}33 \\
34 \\
35 \\
36\end{array}$ & $1: 400$ & $\begin{array}{l}+ \\
+ \\
+ \\
+\end{array}$ & $\begin{array}{l}+ \\
\operatorname{tr} \\
\pm \\
+\end{array}$ & $\begin{array}{r}0 \\
0 \\
0 \\
\pm\end{array}$ & $\begin{array}{l}+ \\
+ \\
+ \\
+\end{array}$ & $\begin{array}{l}+ \\
+ \\
+ \\
+\end{array}$ & $\begin{array}{l} \pm \\
\pm \\
\pm \\
\pm\end{array}$ & $\begin{array}{ll}\text { Moderately positive, } & 2+ \\
\text { Strongly positive, } & 4+ \\
\text { Strongly positive, } & 3+ \\
\text { Negative, } & -\end{array}$ \\
\hline
\end{tabular}

* Explanation: $0=$ no hemolysis; $\operatorname{tr}$ (trace) $=$ hemolysis up to $50 \% ; \pm=$ hemolysis between $50 \%$ and $100 \% ; f=$ complete hemolysis. 
TABLE 2-Continued

Unmixed Frozen Complement Tested at Intervals of a Week

\begin{tabular}{|c|c|c|c|c|c|c|c|c|c|c|c|c|}
\hline \multirow{3}{*}{$\begin{array}{l}\text { Number } \\
\text { of } \\
\text { Comple- } \\
\text { ment }\end{array}$} & \multirow{3}{*}{$\begin{array}{c}\text { Portions } \\
\text { A-Fresh } \\
\text { B-Frozen }\end{array}$} & \multirow{3}{*}{$\begin{array}{c}\text { Age, } \\
\text { Weeks }\end{array}$} & \multirow{3}{*}{$\begin{array}{c}\text { Number } \\
\text { of } \\
\text { Serumn }\end{array}$} & \multirow{3}{*}{$\begin{array}{l}\text { Dilu- } \\
\text { tion } \\
\text { of } \\
\text { Ambo- } \\
\text { ceptor }\end{array}$} & \multicolumn{6}{|c|}{ Readings } & \multirow{3}{*}{\multicolumn{2}{|c|}{ Aesults }} \\
\hline & & & & & \multicolumn{3}{|c|}{$\begin{array}{c}\text { Antigen } \\
\text { Tubes }\end{array}$} & \multicolumn{3}{|c|}{$\begin{array}{c}\text { Control } \\
\text { Tubes }\end{array}$} & & \\
\hline & & & & & 1 & 2 & 3 & $1^{\prime}$ & $2^{\prime}$ & $3^{\prime}$ & & \\
\hline 11 & B & 1 & $\begin{array}{l}33 \\
34 \\
35 \\
36\end{array}$ & $1: 400$ & $\begin{array}{l}+ \\
+ \\
+ \\
+\end{array}$ & $\begin{array}{l} \pm \\
\frac{ \pm}{t r} \\
\frac{ \pm}{+}\end{array}$ & $\begin{array}{l}0 \\
0 \\
0 \\
\pm\end{array}$ & $\begin{array}{l}+ \\
+ \\
+ \\
+\end{array}$ & $\begin{array}{l}+ \\
+ \\
+ \\
+\end{array}$ & $\begin{array}{l} \pm \\
\frac{ \pm}{ \pm} \\
\pm\end{array}$ & $\begin{array}{l}\text { Strongly positive, } \\
\text { Strongly positive, } \\
\text { Strongly positive, } \\
\text { Negative, }\end{array}$ & $\begin{array}{l}3+ \\
4+ \\
3+ \\
-\end{array}$ \\
\hline 11 & $\mathbf{B}$ & 2 & $\begin{array}{l}38 \\
31 \\
35 \\
36\end{array}$ & $1: 400$ & $\begin{array}{l}+ \\
+ \\
+ \\
+\end{array}$ & $\begin{array}{l}+ \\
\operatorname{tr} \\
\operatorname{tr} \\
+\end{array}$ & $\begin{array}{l}0 \\
0 \\
0 \\
\pm\end{array}$ & $\begin{array}{l}+ \\
+ \\
+ \\
+\end{array}$ & $\begin{array}{l}+ \\
+ \\
+ \\
+\end{array}$ & $\begin{array}{l} \pm \\
\pm \\
\text { tr } \\
\pm\end{array}$ & $\begin{array}{l}\text { Moderately positive, } \\
\text { Strongly positive, } \\
\text { Strongly positive, } \\
\text { Negative, }\end{array}$ & $\begin{array}{l}2+ \\
4+ \\
3+ \\
+\end{array}$ \\
\hline 11 & B & 3 & $\begin{array}{l}33 \\
34 \\
35 \\
36\end{array}$ & $1: 400$ & $\begin{array}{l}+ \\
+ \\
+ \\
+\end{array}$ & $\begin{array}{l}\operatorname{tr} \\
\pm \\
\operatorname{tr} \\
+\end{array}$ & $\begin{array}{c}0 \\
0 \\
0 \\
\text { tr }\end{array}$ & $\begin{array}{l}+ \\
+ \\
+ \\
+\end{array}$ & $\begin{array}{l}+ \\
+ \\
+ \\
+\end{array}$ & $\begin{array}{c}0 \\
t r \\
0 \\
t r\end{array}$ & $\begin{array}{l}\text { Moderately positive, } \\
\text { Moderately positive, } \\
\text { Moderately positive, } \\
\text { Negative, }\end{array}$ & $\begin{array}{l}2+ \\
2+ \\
2+ \\
-\end{array}$ \\
\hline 11 & B & 4 & $\begin{array}{l}33 \\
34 \\
35 \\
36\end{array}$ & $1: 400$ & $\begin{array}{l}+ \\
+ \\
+ \\
+\end{array}$ & $\begin{array}{c}0 \\
0 \\
t r \\
+\end{array}$ & $\begin{array}{l}0 \\
0 \\
0 \\
0\end{array}$ & $\begin{array}{l}+ \\
+ \\
+ \\
+\end{array}$ & $\begin{array}{l}\operatorname{tr} \\
\pm \\
+ \\
+\end{array}$ & $\begin{array}{l}0 \\
0 \\
0 \\
0\end{array}$ & $\begin{array}{l}\text { Weakly positive, } \\
\text { Moderately positive, } \\
\text { Moderately positive, } \\
\text { Negative, }\end{array}$ & $\begin{array}{l}1+ \\
2+ \\
2+ \\
-\end{array}$ \\
\hline 12 & $\mathbf{A}$ & 0 & $\begin{array}{l}38 \\
24 \\
35 \\
36\end{array}$ & $1: 400$ & $\begin{array}{l}+ \\
+ \\
+ \\
+\end{array}$ & $\begin{array}{l}+ \\
\operatorname{tr} \\
\pm \\
t\end{array}$ & $\begin{array}{r}0 \\
0 \\
0 \\
\pm\end{array}$ & $\begin{array}{l}+ \\
+ \\
+\end{array}$ & $\begin{array}{l}+ \\
+ \\
+ \\
+\end{array}$ & $\begin{array}{l} \pm \\
\pm \\
\pm \\
\pm\end{array}$ & $\begin{array}{l}\text { Moderately positive, } \\
\text { Strongly positive, } \\
\text { Strongly positive, } \\
\text { Negative, }\end{array}$ & $\begin{array}{l}2+ \\
4+ \\
3+ \\
-\end{array}$ \\
\hline 13 & $\mathbf{A}$ & 0 & $\begin{array}{l}37 \\
38 \\
39 \\
40\end{array}$ & $1: 400$ & $\frac{ \pm}{ \pm}$ & $\begin{array}{l}0 \\
0 \\
0 \\
+\end{array}$ & $\begin{array}{c}0 \\
0 \\
0 \\
\mathrm{tr}\end{array}$ & $\begin{array}{l}+ \\
+ \\
+ \\
+\end{array}$ & $\begin{array}{l}+ \\
+ \\
+ \\
+\end{array}$ & $\begin{array}{l}\operatorname{tr} \\
\operatorname{tr} \\
\operatorname{tr} \\
\mathrm{tr}\end{array}$ & $\begin{array}{l}\text { Strongly positive, } \\
\text { Strongly positive, } \\
\text { Strongly positive, } \\
\text { Negative, }\end{array}$ & $\begin{array}{l}5+ \\
5+ \\
5+ \\
-\end{array}$ \\
\hline 13 & $\mathbf{B}$ & 1 & $\begin{array}{l}37 \\
38 \\
39 \\
40\end{array}$ & $1: 400$ & $\begin{array}{l}\operatorname{tr} \\
\operatorname{tr} \\
\operatorname{tr} \\
+\end{array}$ & $\begin{array}{l}\mathbf{0} \\
\mathbf{0} \\
0 \\
+\end{array}$ & $\begin{array}{c}0 \\
0 \\
0 \\
\operatorname{tr}\end{array}$ & $\begin{array}{l}+ \\
+ \\
+ \\
+\end{array}$ & $\begin{array}{l}+ \\
+ \\
+ \\
+\end{array}$ & $\begin{array}{l}0 \\
\operatorname{tr} \\
\operatorname{tr} \\
\operatorname{tr}\end{array}$ & $\begin{array}{l}\text { Strongly positive, } \\
\text { Strongly positive, } \\
\text { Strongly positive, } \\
\text { Negative, }\end{array}$ & $\begin{array}{l}5+ \\
6+ \\
6+ \\
-\end{array}$ \\
\hline 13 & $\mathbf{B}$ & 2 & $\begin{array}{l}37 \\
38 \\
39 \\
40\end{array}$ & $1: 400$ & $\begin{array}{l}\mathrm{tr} \\
\pm \\
\mathrm{tr} \\
+\end{array}$ & $\begin{array}{l}0 \\
0 \\
0 \\
+\end{array}$ & $\begin{array}{c}0 \\
0 \\
0 \\
\operatorname{tr}\end{array}$ & $\begin{array}{l}+ \\
+ \\
+ \\
+\end{array}$ & $\begin{array}{l}+ \\
+ \\
+ \\
+\end{array}$ & $\begin{array}{l}0 \\
\operatorname{tr} \\
\operatorname{tr} \\
\operatorname{tr}\end{array}$ & $\begin{array}{l}\text { Strongly positive, } \\
\text { Strongly positive, } \\
\text { Strongly positive, } \\
\text { Negative, }\end{array}$ & $\begin{array}{l}5+ \\
5+ \\
6+ \\
-\end{array}$ \\
\hline 13 & B & 3 & $\begin{array}{l}37 \\
38 \\
39 \\
40\end{array}$ & $1: 400$ & $\begin{array}{l} \pm \\
\operatorname{tr} \\
0 \\
+\end{array}$ & $\begin{array}{l}0 \\
0 \\
0 \\
+\end{array}$ & $\begin{array}{c}0 \\
0 \\
0 \\
\operatorname{tr}\end{array}$ & $\begin{array}{l}+ \\
+ \\
+ \\
+\end{array}$ & $\begin{array}{l}+ \\
+ \\
+ \\
+\end{array}$ & $\begin{array}{c}0 \\
0 \\
0 \\
\operatorname{tr}\end{array}$ & $\begin{array}{l}\text { Strongly positive, } \\
\text { Strongly positive, } \\
\text { Strongly positive, } \\
\text { Negative, }\end{array}$ & $\begin{array}{l}4+ \\
5+ \\
6+ \\
-\end{array}$ \\
\hline 13 & B & 4 & $\begin{array}{l}37 \\
38 \\
39 \\
40\end{array}$ & $1: 400$ & $\begin{array}{l} \pm \\
\pm \\
\operatorname{tr} \\
+\end{array}$ & $\begin{array}{r}0 \\
0 \\
0 \\
\pm\end{array}$ & $\begin{array}{l}0 \\
0 \\
0 \\
0\end{array}$ & $\begin{array}{l}+ \\
+ \\
+ \\
+\end{array}$ & $\frac{ \pm}{ \pm}$ & $\begin{array}{l}0 \\
0 \\
0 \\
0\end{array}$ & $\begin{array}{l}\text { Strongly positive, } \\
\text { Strongly positive, } \\
\text { Strongly positive, } \\
\text { Negative, }\end{array}$ & $\begin{array}{l}3+ \\
3+ \\
4+ \\
-\end{array}$ \\
\hline 16 & A & 0 & $\begin{array}{l}37 \\
38 \\
39 \\
40\end{array}$ & $1: 400$ & $\begin{array}{l}+ \\
\pm \\
\pm \\
+\end{array}$ & $\begin{array}{l}0 \\
0 \\
0 \\
+\end{array}$ & $\begin{array}{r}0 \\
0 \\
0 \\
\pm\end{array}$ & $\begin{array}{l}+ \\
+ \\
+ \\
+\end{array}$ & $\begin{array}{l}+ \\
+ \\
+ \\
+\end{array}$ & $\begin{array}{l} \pm \\
\pm \\
\pm \\
\pm\end{array}$ & $\begin{array}{l}\text { Strongly positive, } \\
\text { Strongly positive, } \\
\text { Strongly positive, } \\
\text { Negative, }\end{array}$ & $\begin{array}{l}5+ \\
6+ \\
6+ \\
-\end{array}$ \\
\hline 14 & A. & b & $\begin{array}{l}41 \\
42 \\
43 \\
44\end{array}$ & $1: 400$ & $\begin{array}{l}+ \\
+ \\
+ \\
+\end{array}$ & $\begin{array}{c}\text { tr } \\
0 \\
\text { tr } \\
+\end{array}$ & $\begin{array}{l}0 \\
0 \\
0 \\
\pm \\
\end{array}$ & $\begin{array}{l}+ \\
+ \\
+ \\
+\end{array}$ & $\begin{array}{l}+ \\
+ \\
t \\
+\end{array}$ & $\begin{array}{l} \pm \\
\pm \\
\pm\end{array}$ & $\begin{array}{l}\text { Strongly positive, } \\
\text { Strongly positive, } \\
\text { Strongly positive, } \\
\text { Negative, }\end{array}$ & $\begin{array}{l}4+ \\
5+ \\
4+\end{array}$ \\
\hline 14 & $\mathbf{B}$ & 1 & $\begin{array}{l}41 \\
42 \\
43 \\
44\end{array}$ & $1: 400$ & $\begin{array}{l}+ \\
+ \\
+ \\
+ \\
+\end{array}$ & $\begin{array}{c}\operatorname{tr} \\
0 \\
\operatorname{tr} \\
+\end{array}$ & $\begin{array}{l}0 \\
0 \\
0 \\
\pm\end{array}$ & $\begin{array}{l}+ \\
+ \\
+ \\
+\end{array}$ & $\begin{array}{l}+ \\
+ \\
+ \\
+\end{array}$ & 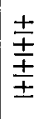 & $\begin{array}{l}\text { Strongly positive, } \\
\text { Strongly positive, } \\
\text { Strongly positive, } \\
\text { Negative, }\end{array}$ & $\begin{array}{l}4+ \\
5+ \\
4+\end{array}$ \\
\hline
\end{tabular}


TABLE 2-Continued

Unmixed Frozen Complement Tested at Intervals of a Week

\begin{tabular}{|c|c|c|c|c|c|c|c|c|c|c|c|c|}
\hline \multirow{3}{*}{$\begin{array}{l}\text { Number } \\
\text { of } \\
\text { Comple } \\
\text { ment }\end{array}$} & \multirow{3}{*}{$\mid \begin{array}{c}\text { Portions } \\
\Lambda \text {-Fresh } \\
\text { B-Frozer }\end{array}$} & \multirow{3}{*}{$\begin{array}{c}\text { Age, } \\
\text { Weeks }\end{array}$} & \multirow{3}{*}{$\begin{array}{c}\text { Number } \\
\text { of } \\
\text { Serum }\end{array}$} & \multirow{3}{*}{$\begin{array}{l}\text { Dilu- } \\
\text { tion } \\
\text { of } \\
\text { Ambo- } \\
\text { ceptor }\end{array}$} & \multicolumn{6}{|c|}{ Readings } & \multirow{3}{*}{\multicolumn{2}{|c|}{ Aesults }} \\
\hline & & & & & \multicolumn{3}{|c|}{$\begin{array}{c}\text { Antigen } \\
\text { T'ubes }\end{array}$} & \multicolumn{3}{|c|}{$\begin{array}{c}\text { Control } \\
\text { rubes }\end{array}$} & & \\
\hline & & & & & 1 & 2 & 3 & $1^{\prime}$ & 2 & 3 & & \\
\hline 14 & B & 2 & $\begin{array}{l}41 \\
42 \\
43 \\
44\end{array}$ & $1: 400$ & $\begin{array}{l}+ \\
+ \\
+ \\
+\end{array}$ & $\begin{array}{l}\operatorname{tr} \\
\operatorname{tr} \\
\operatorname{tr} \\
+\end{array}$ & $\begin{array}{l}0 \\
0 \\
0 \\
\pm\end{array}$ & $\begin{array}{l}+ \\
+ \\
+ \\
+\end{array}$ & $\begin{array}{l}+ \\
+ \\
+ \\
+\end{array}$ & $\begin{array}{l} \pm \\
\pm \\
\pm \\
\pm\end{array}$ & $\begin{array}{l}\text { Strongly positive, } \\
\text { Strongly positive, } \\
\text { Strongly positive, } \\
\text { Negative, }\end{array}$ & $\begin{array}{l}4+ \\
4+ \\
4+ \\
-\end{array}$ \\
\hline 14 & B & 3 & $\begin{array}{l}41 \\
42 \\
43 \\
44\end{array}$ & $1: 400$ & $\begin{array}{l}+ \\
+ \\
+ \\
+\end{array}$ & $\begin{array}{c}\operatorname{tr} \\
0 \\
\operatorname{tr} \\
+\end{array}$ & $\begin{array}{c}0 \\
0 \\
0 \\
\operatorname{tr}\end{array}$ & $\begin{array}{l}+ \\
+ \\
+ \\
+\end{array}$ & $\begin{array}{l}+ \\
+ \\
+ \\
+\end{array}$ & $\begin{array}{l}\operatorname{tr} \\
\operatorname{tr} \\
\operatorname{tr} \\
\operatorname{tr}\end{array}$ & $\begin{array}{l}\text { Strongly positive, } \\
\text { Strongly positive, } \\
\text { Strongly positive, } \\
\text { Negative, }\end{array}$ & $\begin{array}{l}3+ \\
4+ \\
3+ \\
-\end{array}$ \\
\hline 14 & B & 4 & $\begin{array}{l}41 \\
42 \\
43 \\
44\end{array}$ & $1: 400$ & $\begin{array}{l}+ \\
\pm \\
+ \\
+\end{array}$ & $\begin{array}{l}0 \\
0 \\
0 \\
+\end{array}$ & $\begin{array}{l}0 \\
0 \\
0 \\
0\end{array}$ & $\begin{array}{l}+ \\
+ \\
+ \\
+\end{array}$ & $\begin{array}{l}+ \\
+ \\
+ \\
+\end{array}$ & $\begin{array}{l}0 \\
0 \\
0 \\
0\end{array}$ & $\begin{array}{l}\text { Strongly positive, } \\
\text { Strongly positive, } \\
\text { Strongly positjve, } \\
\text { Negative, }\end{array}$ & $\begin{array}{l}3+ \\
4+ \\
3+ \\
-\end{array}$ \\
\hline 16 & A & 0 & $\begin{array}{l}41 \\
42 \\
43 \\
44\end{array}$ & $1: 400$ & $\begin{array}{l}+ \\
+ \\
+ \\
+\end{array}$ & $\begin{array}{c}\operatorname{tr} \\
0 \\
0 \\
+\end{array}$ & $\begin{array}{l}0 \\
0 \\
0 \\
\pm\end{array}$ & $\begin{array}{l}+ \\
+ \\
+ \\
+\end{array}$ & $\begin{array}{l}+ \\
+ \\
+ \\
+\end{array}$ & $\begin{array}{l} \pm \\
\pm \\
\pm \\
\pm\end{array}$ & $\begin{array}{l}\text { Strongly positive, } \\
\text { Strongly positive, } \\
\text { Strongly positive, } \\
\text { Negative, }\end{array}$ & $\begin{array}{l}4+ \\
5+ \\
5+ \\
-\end{array}$ \\
\hline 15 & A & 0 & $\begin{array}{l}45 \\
46 \\
47 \\
48\end{array}$ & $1: 400$ & $\begin{array}{l} \pm \\
\pm \\
+ \\
+\end{array}$ & $\begin{array}{l}0 \\
0 \\
0 \\
+\end{array}$ & $\begin{array}{c}0 \\
0 \\
0 \\
\pm\end{array}$ & $\begin{array}{l}+ \\
t \\
+ \\
+\end{array}$ & $\begin{array}{l}+ \\
+ \\
+ \\
+\end{array}$ & $\begin{array}{l} \pm \\
\pm \\
\pm \\
\pm\end{array}$ & $\begin{array}{l}\text { Strongly positive, } \\
\text { Strongly positive, } \\
\text { Strongly positive, } \\
\text { Negative, }\end{array}$ & $\begin{array}{l}6+ \\
6+ \\
5+ \\
-\end{array}$ \\
\hline 15 & $\mathrm{~B}$ & 1 & $\begin{array}{l}45 \\
46 \\
47 \\
48\end{array}$ & $1: 400$ & $\begin{array}{l}\operatorname{tr} \\
\pm \\
\pm\end{array}$ & $\begin{array}{l}0 \\
0 \\
0 \\
+\end{array}$ & $\begin{array}{l}0 \\
0 \\
0 \\
\pm \\
\end{array}$ & $\begin{array}{l}+ \\
+ \\
+ \\
+\end{array}$ & $\begin{array}{l}+ \\
+ \\
+ \\
+\end{array}$ & $\begin{array}{l} \pm \\
\pm \\
\pm\end{array}$ & $\begin{array}{l}\text { Strongly positive, } \\
\text { Strongly positive, } \\
\text { Strongly positive, } \\
\text { Negative, }\end{array}$ & $\begin{array}{l}8+ \\
6+ \\
6+ \\
-\end{array}$ \\
\hline 15 & B & 2 & $\begin{array}{l}45 \\
46 \\
47 \\
48\end{array}$ & $1: 400$ & $\begin{array}{l}\frac{ \pm}{ \pm} \\
\frac{+}{+} \\
+\end{array}$ & $\begin{array}{l}0 \\
0 \\
0 \\
+\end{array}$ & $\begin{array}{r}0 \\
0 \\
0 \\
\pm\end{array}$ & $\begin{array}{l}+ \\
+ \\
+ \\
+\end{array}$ & $\begin{array}{l}+ \\
+ \\
+ \\
+\end{array}$ & $\begin{array}{l} \pm \\
\pm \\
\pm\end{array}$ & $\begin{array}{l}\text { Strongly positive, } \\
\text { Strongly positive, } \\
\text { Strongly positive, } \\
\text { Negative, }\end{array}$ & $\begin{array}{l}6+ \\
6+ \\
5+ \\
-\end{array}$ \\
\hline 15 & B & 3 & $\begin{array}{l}45 \\
46 \\
47 \\
48\end{array}$ & $1: 400$ & $\begin{array}{l} \pm \\
\pm \\
+ \\
+\end{array}$ & $\begin{array}{l}0 \\
0 \\
0 \\
+\end{array}$ & $\begin{array}{c}0 \\
0 \\
0 \\
\text { tr }\end{array}$ & $\begin{array}{l}+ \\
+ \\
+ \\
+\end{array}$ & $\begin{array}{l}+ \\
+ \\
+ \\
+\end{array}$ & $\begin{array}{l}\operatorname{tr} \\
\mathrm{tr} \\
\mathrm{tr} \\
\mathrm{tr}\end{array}$ & $\begin{array}{l}\text { Strongly positive, } \\
\text { Strongly positive, } \\
\text { Strongly positive, } \\
\text { Negative, }\end{array}$ & $\begin{array}{l}5+ \\
5+ \\
4+ \\
-\end{array}$ \\
\hline 15 & $\mathbf{B}$ & 4 & $\begin{array}{l}45 \\
46 \\
47 \\
48\end{array}$ & $1: 400$ & $\begin{array}{c} \pm \\
t r \\
+ \\
+\end{array}$ & $\begin{array}{l}0 \\
0 \\
0 \\
+\end{array}$ & $\begin{array}{l}0 \\
0 \\
0 \\
0\end{array}$ & $\begin{array}{l}+ \\
+ \\
+ \\
+\end{array}$ & $\begin{array}{l}+ \\
+ \\
+ \\
+\end{array}$ & $\begin{array}{l}0 \\
0 \\
0 \\
0\end{array}$ & $\begin{array}{l}\text { Strongly positive, } \\
\text { Strongly positive, } \\
\text { Strongly positive, } \\
\text { Negative, }\end{array}$ & $\begin{array}{l}4+ \\
5+ \\
3+ \\
-\end{array}$ \\
\hline 16 & A & 0 & $\begin{array}{l}45 \\
46 \\
47 \\
48\end{array}$ & $1: 400$ & $\frac{ \pm}{ \pm}$ & $\begin{array}{l}\hat{v} \\
0 \\
0 \\
+\end{array}$ & $\begin{array}{c}0 \\
0 \\
0 \\
\pm\end{array}$ & $\begin{array}{l}+ \\
+ \\
+ \\
+\end{array}$ & $\begin{array}{l}+ \\
+ \\
+ \\
+\end{array}$ & $\frac{ \pm}{ \pm}$ & $\begin{array}{l}\text { Strongly positive, } \\
\text { Strongly positive, } \\
\text { Strongly positive, } \\
\text { Negative, }\end{array}$ & $\begin{array}{l}6+ \\
6+ \\
5+ \\
+\end{array}$ \\
\hline
\end{tabular}

were almost identical with those given by the fresh complement. After the frozen complement was more than 2 weeks old fixability and hemolytic power gradually decreased; that this decrease in fixability and hemolytic power was not due to changes in the human serums tested is shown by the results given by control complements 12 and 16 , which were nearly identical with the results obtained with the fresh complement 4 weeks previously. 
SUM MARY

Frozen guinea-pig complement one week old, whether composed of the mixed serums of 3 guinea-pigs or of the serum from one guineapig usually gave a little stronger positive results with the Wassermann reaction than did the same complement while fresh. At the age of 2 weeks the frozen complement gave results that were nearly identical with the results obtained with the fresh complements. After 2 weeks the frozen complement gradually lost strength which seemed to be more rapid in mild weather than in very cold weather. (May 10, 1919.) 